\title{
Unicystic Ameloblastoma in a Young Female: A Case Report and Review of Literature
}

\author{
${ }^{1}$ Sham Kishor Kanneppady, ${ }^{2}$ Santosh B Sakri, ${ }^{3}$ Nanditha S, ${ }^{4}$ Laxmikanth Chatra, ${ }^{5}$ Prashanth Shenoy K \\ ${ }^{1}$ Lecturer, Department of Oral Medicine and Radiology, Faculty of Dentistry, AIMST University, Malaysia \\ 2Lecturer, Department of Community Dentistry, Faculty of Dentistry, AIMST University, Malaysia \\ ${ }^{3}$ P rincipal, Lecturer, Department of Periodontology, Faculty of Dentistry, AIMST University, Malaysia \\ ${ }^{4}$ Senior Professor and Head, Department of Oral Medicine and Radiology, Yenepoya Dental College, Mangalore, Karnataka, India \\ ${ }^{5}$ Professor and Postgraduate Guide, Department of Oral Medicine and Radiology, Yenepoya Dental College, Mangalore, Karnataka, India
}

Correspondence: Sham Kishor Kanneppady, Lecturer, Department of Oral Medicine and R adiology, Faculty of Dentistry, AIMST University, Semeling 08100 Bedong, Kedah Darul Aman, Malaysia, Phone: 006-0103719885, e-mail: drsham.omr@gmail.com

\section{ABSTRACT}

Ameloblastoma is an archetype of a true neoplasm of odontogenic origin. Most ameloblastomas have been classically described as a multilocular cyst-like lesion of the jaw. The unicystic variant is far less frequent and has been reported in only $6 \%$ of ameloblastomas. The presented patient is an 18-year-old female, diagnosed with this distinct entity which was treated with hemimandibulectomy, followed by reconstruction.

Keywords: Ameloblastoma, Unicystic, Posterior mandible, Reconstruction.

\section{INTRODUCTION}

Odontogenic tumors encompass a broad range of benign and malignant neoplasms arising from odontogenic residues. A meloblastomaisthecommonestbenign tumor, which develops from the cellular elements and dental tissues in their various phases of devel opment. ${ }^{1}$ Itisaclassic exampleof atrueneoplasm of enamel organ type tissue that lacks the potential to undergo differentiation, and hencehas aptly been defined as "unicentric, nonfunctional, intermittentingrowth, anatomically benign and clinically persistent" by Robinson. The earlier term used to describe this neoplasm 'adamantinoma' has been replaced by ameloblastomaas theneoplasm is not associated with hard tissue formation. A meloblastomas are usual ly firstrecognized between the ages of 30 and 50 , being rare in children and old people. ${ }^{2}$ It is generally considered to begender neutral, although itmay be slightly morecommon in men. ${ }^{3} \mathrm{~A}$ bout $80 \%$ form in themandible; of these, $70 \%$ develop in the posterior molar region, and often involve the ramus. Lesions are symptomless until the swelling becomes obtrusive. ${ }^{2}$ A lthough maxillary ameloblastomas are less common, they are potentially lethal, especially when the maxillary sinus is involved or tumor cells invade through bone into the soft tissue. Radiographically, ameloblastoma may cast aunilocular cyst, likeradiolucency or a multilocular image with soap-bubble, honeycomb or tennis-racket pattern. In places, cortices are spared and expanded, and in other regions, they are destroyed; root resorption is a common finding. ${ }^{3}$ A mel oblastomas can broadly be classified based on the site of occurrence and radiographic features as peripheral and intraosseous. Peripheral tumors are odontogenic tumors with the histological characteristics of intraosseous amel oblastoma that occur solely in the soft tissues covering the tooth-bearing parts of the jaws. ${ }^{4,5} \mathrm{M}$ ost of the intraosseous ameloblastomas being multilocular cyst-like lesions, the unicystic variant is far less frequent and has been reported in only $6 \%$ of the amel oblastomas. W epresenta case of large unicystic amel oblastoma in a young female.

\section{CASE REPORT}

A n 18-year-old female presented to Department of Oral M edicine and Radiology, $Y$ enepoya Dental College, M angalore, with the complaint of painless swelling in right lower-third of the face of one month duration, which was gradually increasing in size. She had no difficulty in chewing or swallowing but complained of recurrent paresthesia in her lower lip. There was no history of fever or trauma to the facial region and neither had she performed any deleterious habits in her lifetime. On physical examination, patient was of medium build and appeared clinically normal. A Il vital signs were within the acceptable range on the day of presentation.

Except for mild asymmetry in the right lower region of the face, all other extraoral findings (TM J, lymph nodes and salivary glands) were normal (Fig. 1). The swelling was hard in consistency and extended superoinferiorly from ala-tragal line to $1 \mathrm{~cm}$ below the lower border of the mandible; anteroposteriorly, from right commissure up to the posterior border of ramus. Skin overlying the swelling was normal in color without any dilated superficial veins. Intraorally, full complements of teeth were present for her age and none of the teeth were carious or periodontally compromised. There was no associated trismus. However, vestibular obliteration was evident at lower right 


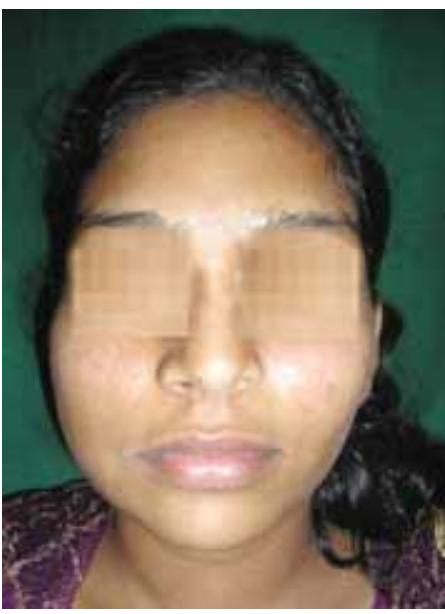

Fig. 1: Mild asymmetry in the right lower region of the face

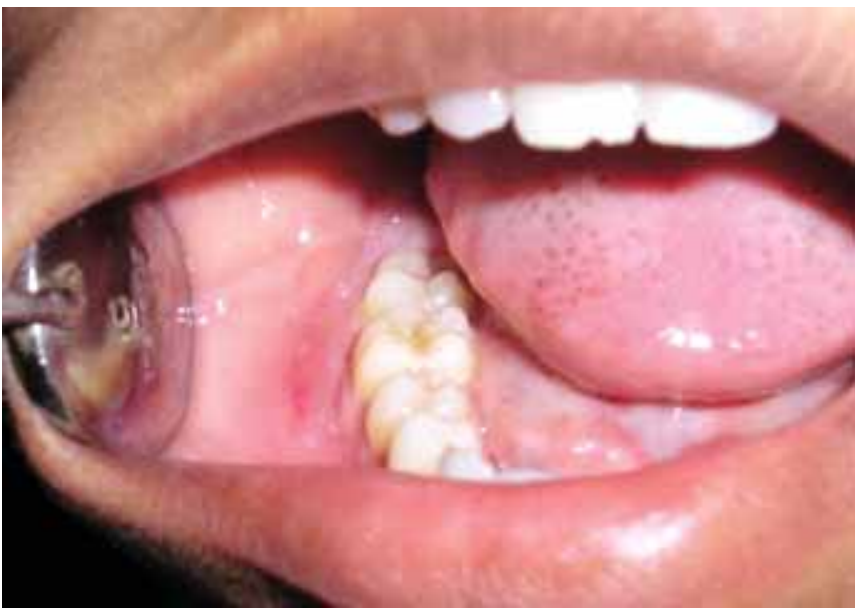

Fig. 2: Vestibular obliteration in mandibular right posterior region

posterior region of 45,46 and 47 with normal overlying mucosa (Fig. 2). On palpation, the swelling was firm in consistency throughout, except in some areas at the lingual aspect where there were definite depressions, probably due to loss of cortical plate. Associated teeth were nonmobile and nontender on percussion. Considering the history and clinical findings, we arrived at a differential diagnosis of either ameloblastoma or dentigerous cyst involving 48.

$\checkmark$ itality test reveal ed delayed response with 46 and 47 ; nonresponsive with 45 . FNAC of the lesion was inconclusive. A wide array of radiographs and CT scan were done to aid in the diagnosis and to determine the extensiveness of the lesion. Intraoral periapical radiograph of the region showed radiolucent lesion starting from apex of 45 , extending posteriorly with root resorption in 45, 46 and 47 (Fig. 3). Occlusal radiograph revealed expanded and thinned cortical plates at mandibular right posterior aspect (Fig. 4). Orthopantomogram was taken, which showed the entire extension of the cystic lesion involving right body and ramus of the mandible (Fig. 5). An impacted tooth at the angle of mandible was seen within the lesion, which could be 48 . Some areas were more radiolucent compared to

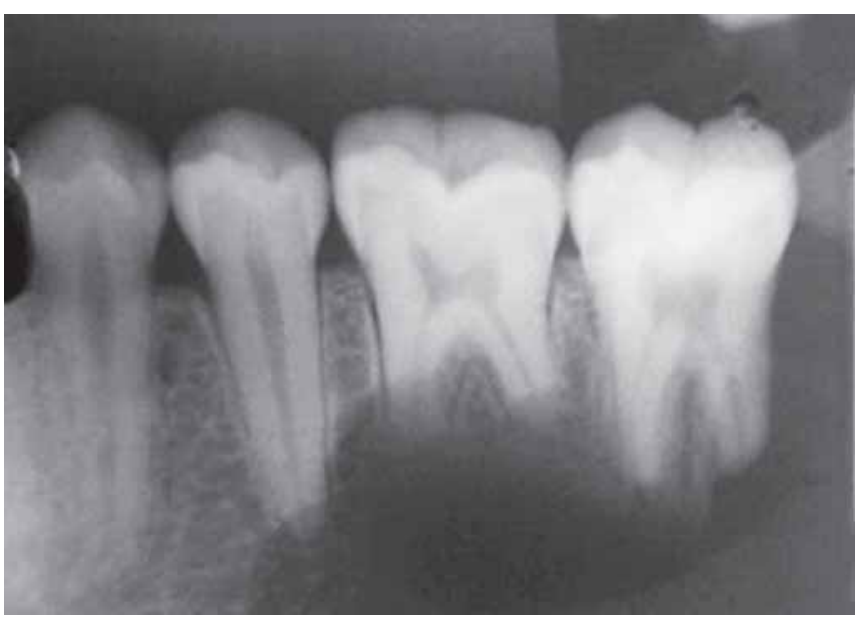

Fig. 3: Intraoral periapical radiograph showing radiolucent lesion and root resorption

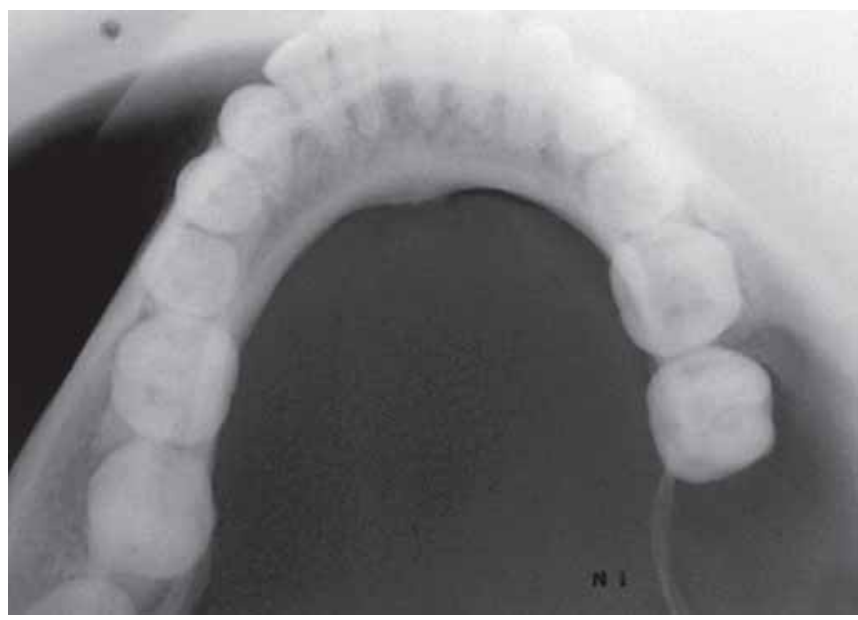

Fig. 4: Occlusal radiograph showing expanded and thinned cortical plates at mandibular right posterior region

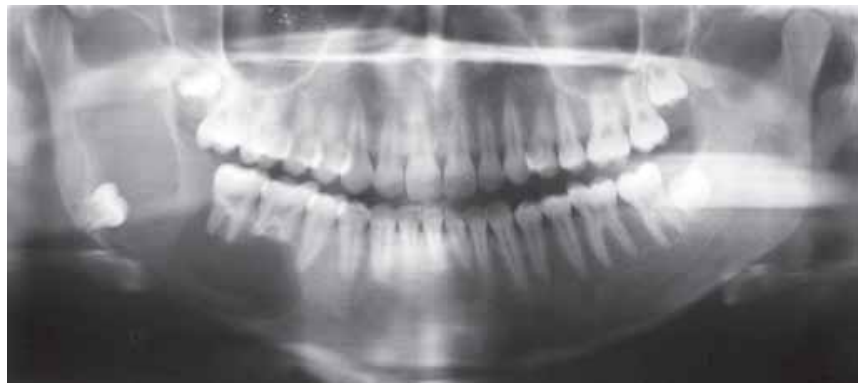

Fig. 5: Orthopantomogram showing the entire cystic lesion, involving 48 in right body and ramus of the mandible

adjacent, which confirmed the clinical finding of loss of cortical plate. CT scan demonstrated expansile cystic lesion involving body and ramus of the mandible with thinning and loss of cortices (Figs 6 and 7).

Patient was taken up for surgery under general anesthesia. Hemimandibulectomy was performed from the region of 44 , retaining the condyle (Fig. 8). The excised specimen had histopathologic features consistent with unilocular amel oblastoma (Fig. 9). Reconstruction was done using rib graft 


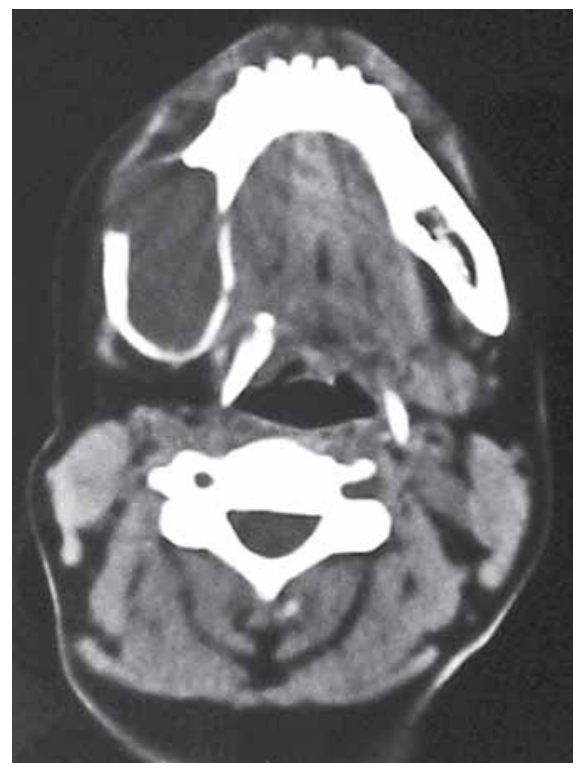

Fig. 6: Axial CT image showing cystic lesion in right mandible with thinning and loss of cortices

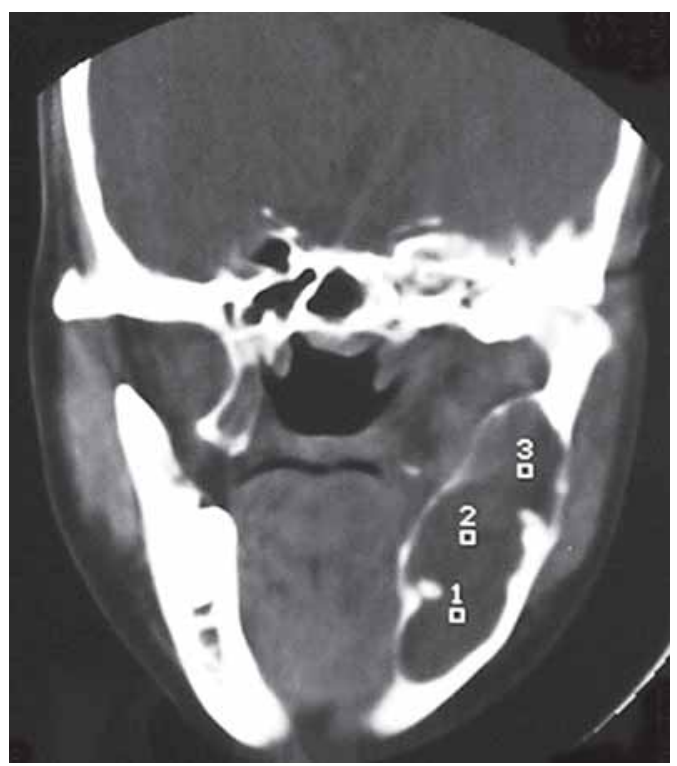

Fig. 7: Coronal CT image showing expansile cystic lesion at the level of ramus

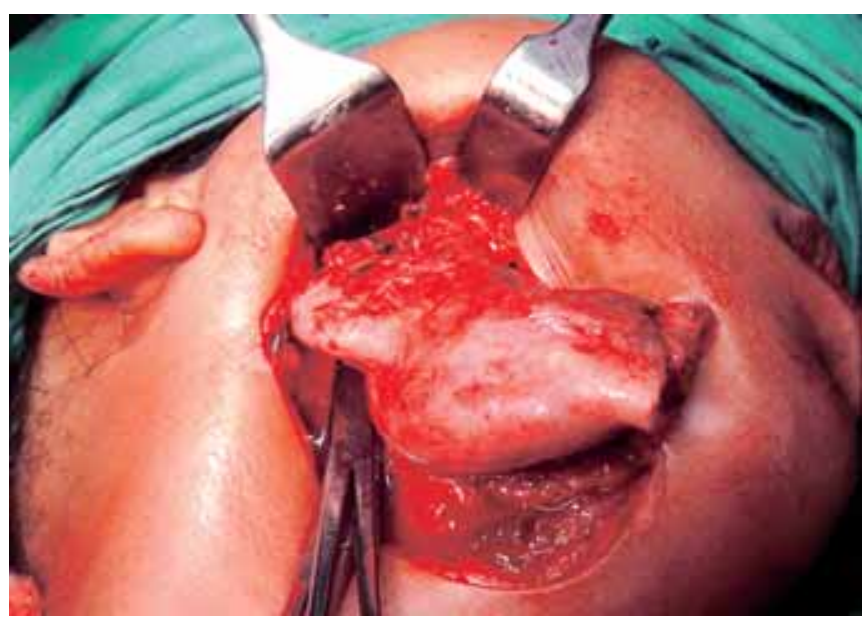

Fig. 8: Hemimandibulectomy being performed

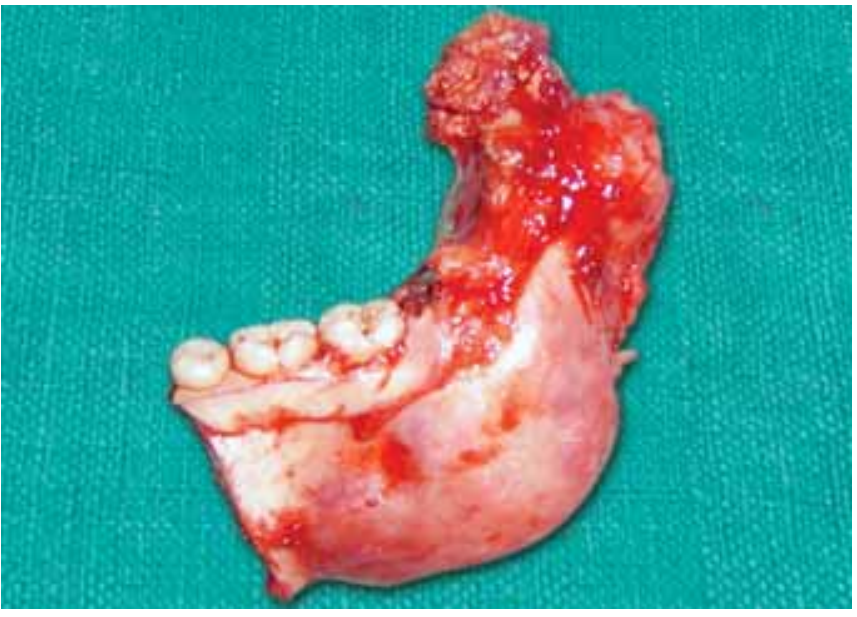

Fig. 9: Resected specimen

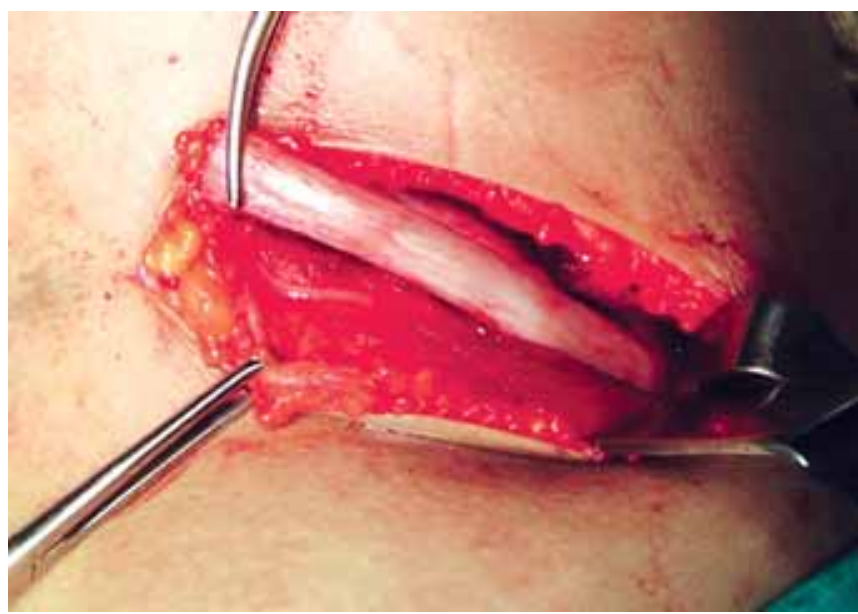

Fig. 10: Rib graft being taken out for reconstruction

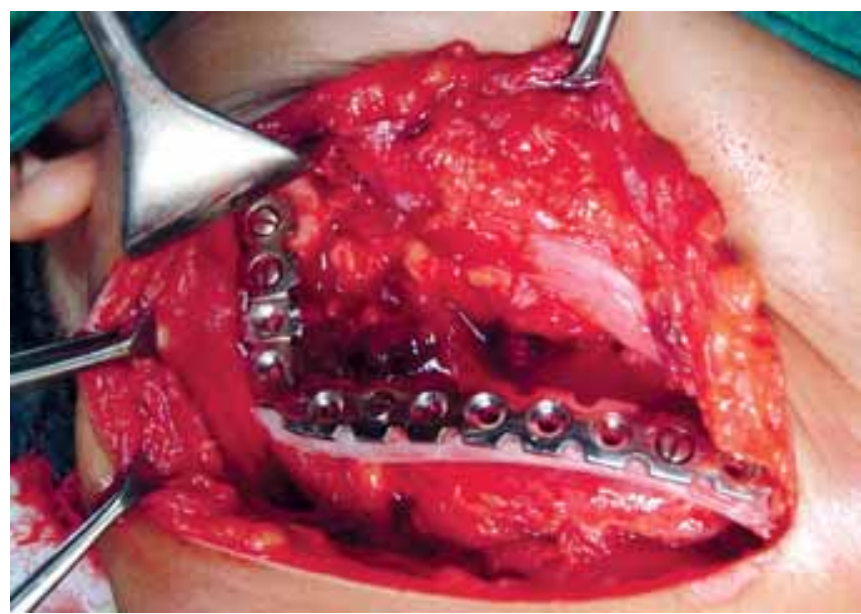

Fig. 11: Rib graft fitted to bone plate is fixed to condyle and body for reconstruction 


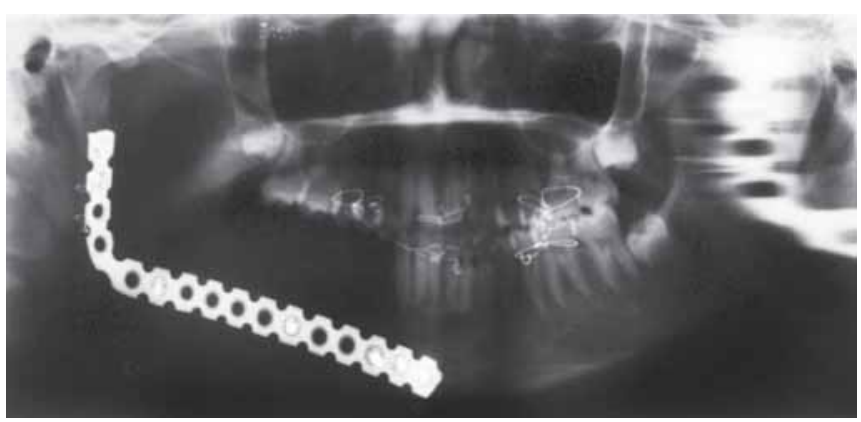

Fig. 12: Postsurgical orthopantomogram showing bone plate fixed in resected area

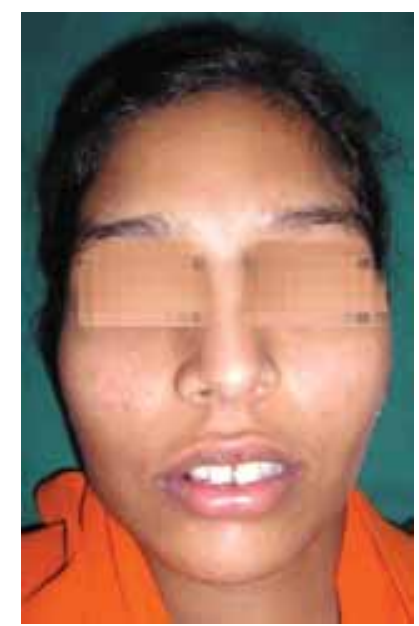

Fig. 13: P hotograph after one week of surgery showing well-maintained facial symmetry

fitted to the stainless steel bone plate (Figs 10 to 12). Facial symmetry was well maintained postsurgically (Fig. 13). One year systematic follow-up did not reveal any recurrence.

\section{DISCUSSION}

Unicystic ameloblastoma (UA) is a distinct entity. It refers to those cystic lesions that show clinical, radiographic or gross features of a mandibular cyst, but on histologic examination shows a typical ameloblastomatous epithelium lining part of the cyst cavity, with or without luminal and/or mural tumor growth. ${ }^{4}$ Robinson and $M$ artinez were the first persons to describe $U A$ in $1977 .{ }^{6,7}$ It is most commonly seen in individuals who are 16 to 20 years of age. Occasionally, lesions occur in younger patients; rarely, they have been found in patients up to the age of $40 .{ }^{8}$ A bout $90 \%$ of the lesions are located in the mandible and between 50 to $80 \%$ of these cases are associated with an impacted tooth. ${ }^{9,10}$ As seen in the present case, mandibular third molars are most commonly implicated with UA . Facial asymmetry due to swelling is the regular presenting feature which infrequently relates with pain. ${ }^{4}$

The radiographic appearance is important in the diagnosis; as it determines whether the lesion is unilocular, a necessary criterion for unicystic ameloblastoma. Lesions are usually well demarcated and may even be corticated. M ost of these cystic tumors enclose the crown of a tooth and mimic dentigerous cyst radiographically. When UA is located in premolar area, the roots of the adjacent teeth may be displaced. ${ }^{8}$

General histological features of UA consist of a dense, uniformly thickened, fibrous connective tissue capsule, surrounding a solitary, large, and fluid-filled lumen. The epithelial lining of the lumen is uniform in thickness and has a slightly hyperchromatic layer of palisaded basal cell, most of which exhibit reverse pol arization of the nucleus. The remaining layers resemble stellate reticulum. However, three distinct histopathologic groups of UA have been documented in the literature. If lesions contain areas in which the epithelium is thickened with papillary projections extending into the lumen, it is said to be intraluminal UA. When the thickened lining penetrates the adjacent capsular tissue, it is termed mural UA . The third variety, plexiforn UA consists of intral uminal nodular projections that contain a netw ork or mesh pattern of epithelium without the distinctive amel oblast-like changes of the basal cell layer. ${ }^{4,8}$

Different types of treatment options have been proposed for the management of ameloblastoma, ranging from curettage to a combination of surgery and radiation therapy. However, curettage is the least desirable of all methods due to its association with a high recurrence rate, and radiation therapy is usual ly not warranted as the lesion is radioresistant. Diagnosis of UA plays a pivotal role in planning the treatment for a patient. This is owed to the fact that the recurrence rate of this lesion is distinctly lower indicating a less aggressive nature of this variant compared to the characteristic ameloblastoma. ${ }^{11}$ Thus, the overall prognosis for $\mathrm{UA}$ is considerably better than the other variants. ${ }^{12}$ In our particul ar case, treatment was planned based on the patient's age, extensiveness of the lesion, site of occurrence and the esthetic concerns thereafter. The challenge in the management of large ameloblastoma of the mandible is not only to excise the tumor completely but also to offer the best reconstruction method to regain facial symmetry. Considering the fact that the presented patient was a young female, esthetic concern of the patient played a major part in the treatment plan. Following hemimandibulectomy, acceptable facial form was restored using rib grafting plated to the resected site. Literature suggests that even U A are associated with $10 \%$ recurrences, ${ }^{8}$ and hence require a systematic follow-up. The presented patient was, abiding to the protocol, followed up for a period of one year during which no complications or signs of recurrence were observed.

\section{ACKNOWLEDGMENTS}

The authors would like to thank the staff and postgraduate students of the Department of Oral and M axillofacial Surgery, $Y$ enepoya Dental College, for their kind hel $p$ with the surgical management photos. 


\section{REFERENCES}

1. Rajendran R, Sivapathasundaram B. Shafer's textbook of oral pathology (5th ed), New Delhi: Elsevier 2006.

2. Cawson RA, Odell EW. Cawson's essentials of oral pathology and oral medicine (7th ed). Churchill Livingstone 2004.

3. Wood NK, Goaz PW. Differential diagnosis of oral and maxillofacial lesions (5th ed). N ew D elhi: M osby, 2006.

4. Ramesh Rakesh S, M anjunath Suraj, Ustad H Tanveer, et al. Unicystic amel oblatoma of the mandible- an unusual case report and review of literature. Head and Neck Oncology 2010;2:1.

5. Chana, Jagdeep S, Y ang-M ing Chang, et al. Segmental mandibul ectomy and immediate free fibula osteoseptocutaneous flap reconstruction with endosteal implants: A $n$ ideal treatment method for mandibular ameloblastoma. Plast Reconstr Surg 2004;113(1):80-87.

6. Srinivasan $\mathrm{H}, \mathrm{A}$ rathy $M$ anohar. U nicystic ameloblastoma of the mandible: A case report. A nnals and E ssence of D entistry 2010; 2(4):75-77.
7. Navarro CM, Principi SM, M assucato EM, Sposto MR. M axillary unicystic ameloblastoma. Dentomaxillofac Radiol 2004;33:60-62.

8. Sapp JP. Contemporary O ral and M axillofacial Pathology (2nd ed) USA : M osby 2004.

9. Philipsen HP, Reichart PA. Unicystic ameloblastoma. Odontogenic tumors and allied lesions. London: Quintessence Pub. Co. Ltd 2004;77-86.

10. Pizer ME, Page DG, Svirsky JA. Thirteen-year follow-up of large recurrent unicystic ameloblastoma of the mandible in a 15-year-old boy. J Oral M axillofac Surg 2002;60:211-15.

11. Kahairi A, A hmad RL, W an I slah L, et al. M anagement of large mandibular ameloblastoma: A case report and literature reviews. A rchives of Orofacial Sciences 2008;3(2):52-55.

12. Li TJ, Wu YT, Y u SF, Y u GY. U nicystic ameloblastoma: A clinicopathological study of 33 Chinese patients. Am J Surg Pathol 2000;24:1385-92. 